\title{
Advanced Marathi Sign Language Recognition using Computer Vision
}

\author{
Amitkumar Shinde \\ Dr. D. Y. Patil SOET \\ Savitribai Phule University \\ Pune-Maharashtra \\ India
}

\author{
Ramesh Kagalkar \\ Dr. D. Y. Patil SOET \\ Savitribai Phule University \\ Pune-Maharashtra \\ India
}

\begin{abstract}
Sign language is a natural language that uses different means of expression for communication in everyday life. As compare to other sign language ISL interpretation has got less attention by researcher. This paper presents an Automatic translation system for gesture of manual alphabets in Marathi sign language. It deals with images of bare hands, which allows the user to interact with the system in a natural way. System provides an opportunity for deaf persons to communicate with normal people without the need of an interpreter. We are going to build a systems and methods for the automatic recognition of Marathi sign language. The first step of this system is to create a database of Marathi Sign Language. Hand segmentation is the most crucial step in every hand gesture recognition system since if we get better segmented output, better recognition rates can be achieved. The proposed system also includes efficient and robust hand segmentation and tracking algorithm to achieve better recognition rates. A large set of samples has been used to recognize 43 isolated words from the Standard Marathi sign language. In proposed system, we intend to recognize some very basic elements of sign language and to translate them to text and vice versa in Marathi language.
\end{abstract}

\section{General Terms}

Image Capturing, Pre-processing, Feature Extraction, Classification, Pattern Recognition/Matching.

\section{Keywords}

Marathi sign language, Marathi alphabets, Hand gesture, Web-camera, HSV image, colour based hand extraction, centre of gravity.

\section{INTRODUCTION}

Sign language is a type of language that uses hand movements, facial expressions and body language to communicate. It is used predominantly by the deaf and people who can hear but cannot speak. But it is also used by some hearing people, most often families and relatives of the deaf, and interpreters who enable the deaf and wider communities to communicate with each other. Sign Language is a structured language where each gesture has some meaning assigned to it used by deaf sign user. Sign language is only the way of communication for deaf sign user. With the help of advanced science and technology many techniques are developed by the researcher to make the deaf people communicate very fluently. Sign Languages (SLs) are the basic means of communication between hearing impaired people. Static morphs of the hands, called postures, together with hand movements, called gestures, and facial expressions form words and sentences in SLs, corresponding to words and sentences in spoken languages.

Imagine you want to have a conversation with a deaf person. Already this may seem a daunting task, especially if you have no idea on how to communicate using sign language. Such is the problem faced by millions of deaf people who are unable to communicate and interact with hearing people. The problem with Deaf peoples are as, they are marginalized in society and are made to feel unimportant and unwanted. How then can we help to improve the quality of life of the deaf community? Information technology is the solution for such problems. In our quest to seek a most natural form of interaction, we have promoted the development of recognition systems, e.g. text and gesture recognition systems. The advancements in information technology thus hold the promise of offering solutions for the deaf to communicate with the hearing world. Furthermore, the cost of computer hardware continues to decrease in price whilst increasing in processing power, thus opening the possibility of building real-time sign language recognition and translation systems. Real-time sign language translation systems will be able to improve communication and allow the deaf community to enjoy full participation in day-to-day interaction and access to information and services. Sign languages all over the world use both static and dynamic gestures, facial expressions and body postures for communication. In our proposed system we are going to implement Marathi sign Language for deaf sign user.

\section{LITERATURE SURVEY}

For the recognition of the sign language a touch screen based approach is developed in [3]. The author tries to recognize the character generated from the screen sensor and transform to speech signal based on a recognition algorithm. In an approach [4] the author suggests in recognizing the hand gesture based on the finger boundary tracing and fingertip detection. The author suggested to Identify the American Sign Language based on the hand gesture passed.

In [5] a computing approach to hand gesture recognition is developed for hearing and speech impaired. Don Pearson in his approach "Visual Communication Systems for the Deaf" [6] presented a two way communication approach, where he proposed the practicality of switched television for both deafto hearing and deaf-to-deaf Communication. In his approach, attention is given to the requirements of picture communication systems, which enable the deaf to communicate over distances using telephone lines.

Towards the development of automated speech recognition for vocally disabled people a system called "BoltayHaath" [6] is developed to recognize "Pakistan Sign Language"(PSL). The BoltayHaath project aims to produce sound matching the accent and pronunciation of the people from the sign symbol passed. A wearing data Glove for vocally disabled is designed, to transform the signed symbols to audible speech signals using gesture recognition. They use the movements of the hand and fingers with sensors to interface with the computer. The system is able to eliminate a major 
communication gap between the vocally disabled with common community.

But BoltayHaath has the limitation of reading only the hand or finger movements neglecting the body action, which is also used to convey message. This gives a limitation to only transform the finger and palm movements for speech transformation. The other limitation that can be seen with BoltayHaath system is the signer could be able to communicate with a normal person but the vice versa is not possible with it.

\section{MARATHI SIGN LANGUAGE}

Each country has its own sign language defined and used over their country. Similarly Marathi Sign Language is the language used by the deaf sign user over India. Marathi sign language alphabets contain the vowels and consonants. Marathi sign Language alphabets are as follows:

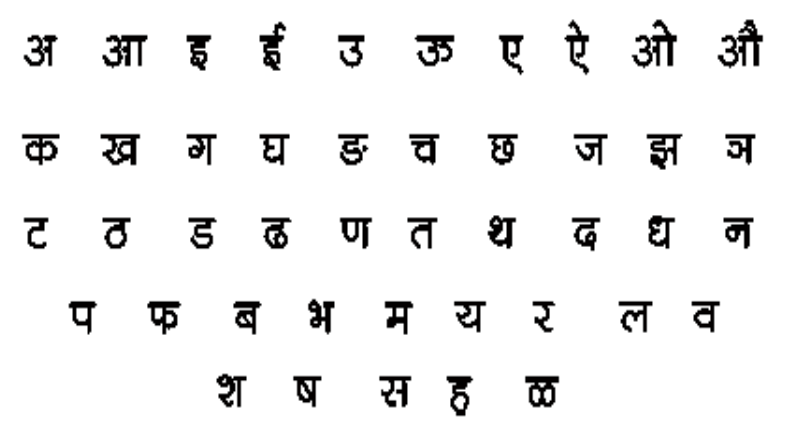

Figure 3.1: Marathi Alphabets

To communicate in sign language requires a specific sign language that can be used as way of communication. Our proposed system is implemented in Marathi sign language. Marathi sign language is Indian sign language used as medium of communication. Figure 3.2 shows the sign language images for corresponding Marathi alphabets. Proposed system is designed to recognize the 43 Marathi sign which consist of vowels and consonants. During the recognition Marathi sign language is translated into corresponding Marathi text and similarly for vice versa.

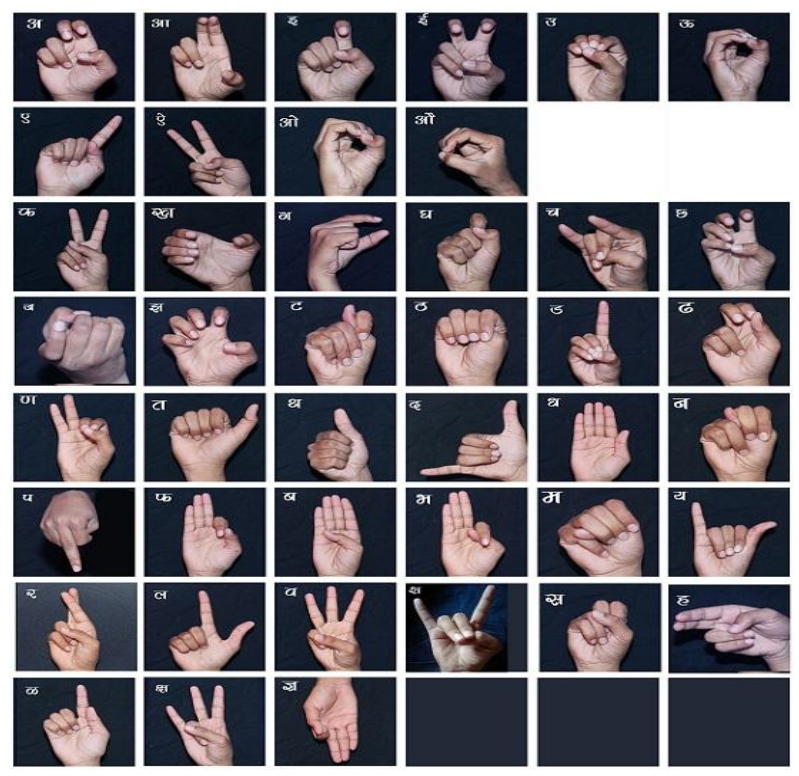

Figure 3.2: Marathi Sign Language images

\section{RELATED WORK}

The proposed system is designed for the deaf person as well as vocal people who are communicating with each other with the help of sign language. The system is helpful for deaf people as well as vocal people when they are migrating in society. The proposed system is us used in both modes i.e. in offline mode and through web camera. In offline mode user can learn how to use sign language and its different signs. In proposed system during translation of sign language to text in offline mode user has to select the input sign image through the database. After selecting the input image then preprocessing and feature extraction is done in that image. After processing the input image is translated to the corresponding text. Similarly during translation of text to sign image the text is entered into the textbox and pre-processing is done. After processing the sign image is displayed on the screen for that text. During the translation of sign language to text and vice versa in offline mode the pattern recognition/matching is done with the help of database which is already present in the database. And during sign language recognition through web camera the hand gesture image is taken from the input device (camera) and that image is processed to find the correct text for corresponding input hand gesture image. This identification of input hand gesture image is the challenging task in the proposed system. The proposed system will identify the correct output from input for which the system is trained. For unknown and wrong input to the system will not give the output to end user. So user has to enter the valid input text or input hand gesture.

\section{PROPOSED SYSTEM}

The proposed system is divided into two parts for sign language recognition:

- Recognition through offline

- Recognition through web camera

In the recognition through offline the user is trained for the Marathi sign language recognition. So using offline mode the deaf sign user as well as vocal people can learn the sign language. The users who are not aware of sign language are trained through this offline mode. In offline recognition user can learn translation of sign language to text as well as translation of text to sign language. In offline mode number of operations such as pre-processing, feature extraction pattern recognition/matching through database is done.

Similarly the user those who are trained in offline recognition can work on the recognition through web camera. In the recognition of sign language through camera the input image is captured through web cam. Then captured image is processed for the recognition. During this process multiple operations are performed on the input image such as image capturing, resizing image, color based detection, noise reduction, center of gravity, and last database comparison.

\section{A. Recognition through offline:}

In the recognition of sign language with offline recognition the user is trained for the particular sign language recognition. The vocal people or the new deaf sign user can use the offline recognition system and can learn sign language. In this recognition the user get aware of the Marathi alphabets as well as the Marathi sign language. The user can learn what the signs are for individual letters also learns its static sign images. Also user can learn how the sentences are formed in the sign language. So this offline method helps to recognition 
of sign language to text and vice versa. The flow of recognition of sign language in offline is as follows:

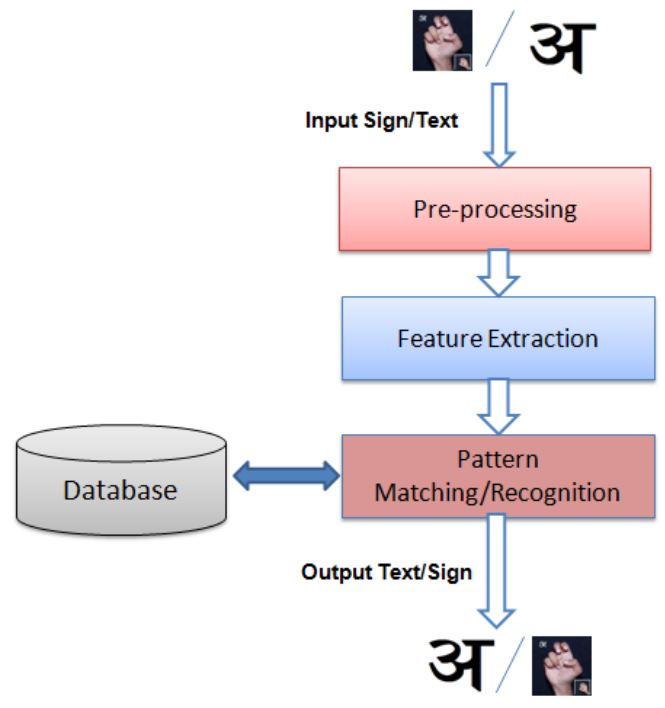

Figure 5.1 Block diagram of translation of sign language to text and text to sign language

\section{i. Input:}

Initially input is taken from user it may be either hand gesture image or Marathi text. The input image is browsed from database and selected as input. And if input is text then it entered through keyboard.

\section{ii. Pre-processing:}

Pre-processing is done during the inputting the text or image. It includes the loading the input to the system. The system takes this input and made it ready for the feature extraction.

\section{iii. Feature Extraction}

During the feature extraction phase the parameters of input image or text are extracted for the recognition. This parameter includes the values stored for the corresponding image or text.

\section{iv. Pattern Matching/Recognition:}

The parameters obtained in the feature extraction phase are compared with database. The database already contains the parameter set for corresponding image or text. So the input parameters are matched with predefines parameters and correct output is recognized.

\section{v. Output:}

The results that are obtained during matching and recognition of input are displayed on the output screen. If input is text then its output will be sign image and if input is sign image then its corresponding output will be text.

\section{B. Recognition through Web-camera:}

When user is successfully trained for the recognition of sign language on offline mode of system the user can go with the sign language recognition using web-camera. The recognition with the web-camera is difficult task because the user has to do proper sign in front of the camera to recognize the correct output. Otherwise system will not work correctly and gives wrong result to user.

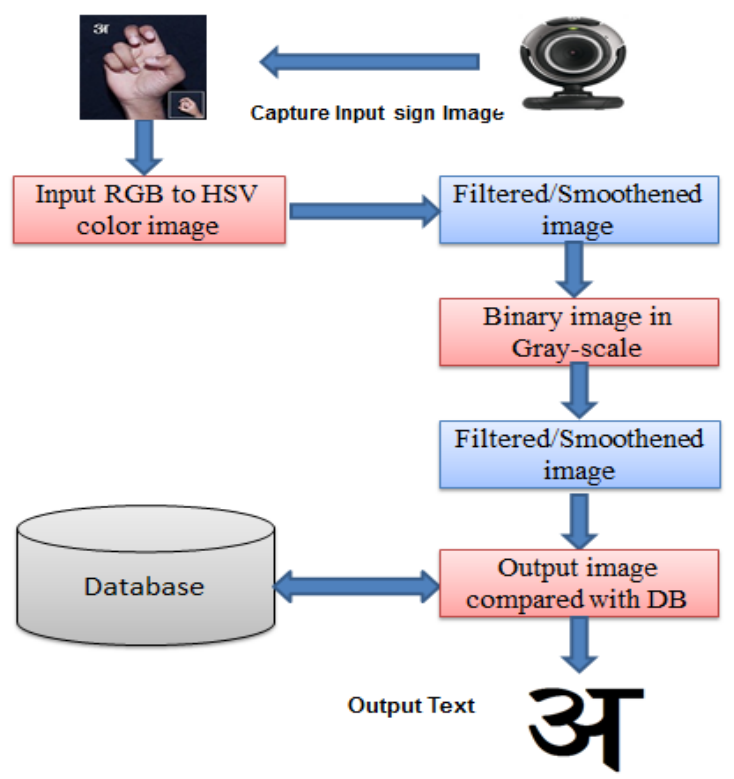

Figure 5.2 Block diagram of Sign language recognition using skin filtering

\section{Capture image from camera:}

Input image is captured from web camera. When user gives the input sign he must give in proper form so the detection and processing of image will be easy.

\section{Resize image:}

As we are considering only static hand shapes we need to capture only hand portion. So resizing of image gives the required image only. Resizing of image reduce processing time of system and perform actions only on required area.

\section{Color based hand extraction:}

In color based hand detection input image is taken which is captured from camera. Initially input image is RGB. So that image is converted to HSV image. Then this HSV image is filtered and smoothened and finally we get image which comprises of only skin colored pixels. This image is binary image in gray scale. Biggest linked skin colored pixels is considered by BLOB i.e. binary linked object. And we get final output which is compared with database. With the help of following formulas input image is converted to HSV image.

$$
\begin{gathered}
f(x)=\left\{\begin{array}{cc}
60\left(\frac{G-B}{\delta}\right) & \text { if } M A X=R \\
60\left(\frac{B-R}{\delta}+2\right) & \text { if } M A X=G \\
60\left(\frac{R-G}{\delta}+4\right) & \text { if } M A X=B \\
\text { not defined } & \text { if } M A X=0
\end{array}\right. \\
f(x)= \begin{cases}\frac{\delta}{M A X} & \text { if } M A X \neq 0 \\
0 & \text { if } M A X=0\end{cases}
\end{gathered}
$$


Where $\delta=($ MAX -MIN), MAX $=\max (\mathrm{R}, \mathrm{G}, \mathrm{B})$ and $\mathrm{MIN}=\min (\mathrm{R}, \mathrm{G}, \mathrm{B})$

\section{Reduce noise:}

Noise reduction gives clean and clear image after color based extraction. So the parameters requited for detection are clearly and easily retrieved. In noise reduction we eliminate surrounding like shadow of skin color, wood, dress etc.

\section{Calculate center of gravity:}

Center of gravity will helps us to made hand in proper way in front of camera. Also the detection of hand portion will be easy with center of gravity.

i. Average height of sign determines the average height of the input image. Based on the average height the hand portion is detected. Lesser portion increased speed of processing and overall performance of system.

ii. Centroid of sign is the average co-ordinates of the input image. Centroid is calculated based on X-direction and $\mathrm{Y}$ direction such as $(\mathrm{X} 1, \mathrm{Y} 1),(\mathrm{X} 2, \mathrm{Y} 2),(\mathrm{Xn}, \mathrm{Yn})$. The centroid can be calculated using following formulas:

$$
X c=\frac{\sum_{i=1}^{n} x_{i}}{\text { area }}
$$

Where, $x_{i}$ represents $\mathrm{X}$ co-ordinates of each boundary

$$
Y_{C}=\frac{\sum_{i=1}^{n} y_{i}}{\text { area }}
$$

Where, $y_{i}$ represents $\mathrm{Y}$ co-ordinates of each boundary

$\mathrm{N}$ is the total number of boundary points. The centroid of image is $\left(X c, Y_{C}\right)$.

iii. The Euclidian distance between two points $(\mathrm{X} 1, \mathrm{Y} 1)$ and $(\mathrm{X} 2, \mathrm{Y} 2)$ can be calculated as:

$$
d=\sqrt{\left(x_{2}-x_{1}\right)^{2}+\left(y_{2}-y_{1}\right)^{2}}
$$

And Euclidian distance between centroid and origin is given by

$$
d=\sqrt{x_{c}^{2}+y_{c}^{2}}
$$

\section{Database Comparison/Matching:}

After getting the required parameters from input image the image is compared with database with the help of those parameters. If the input image is matched with the image in the database the output is displayed on the screen. In this way input sign language image is translated into text.

The database will contain the sets of multiple sign images. Pattern matching and pattern recognition is using predefined datasets.

\section{RESULT AND ANALYSIS}

Result and analysis shows the exact working and the terms considered during the execution of the application or system after the completion. For the recognition of Marathi sign language through offline is easy task for the user. The user is trained through the predefined database. The snapshot given below gives the idea about how sign language recognition is done in offline mode.

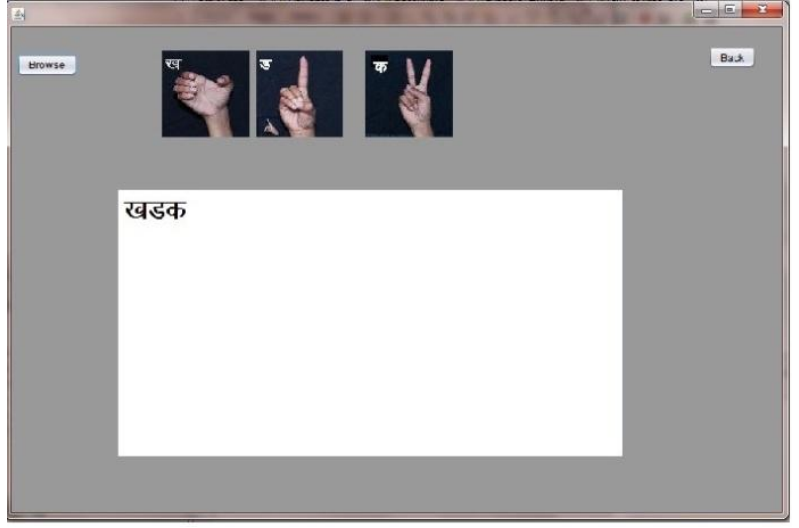

Figure 6.1 translation of sign language to text in offline.

In the recognition of sign language to text the input image is browsed from database and the corresponding text will be displayed in the text box below the image. Likewise user can study individual alphabets as well as sentences of Marathi sign language. The recognition is done using predefined database.

Similarly in the second snapshot the input text is translated to corresponding sign language image. During translation the user has to enter the input text through keyboard. Then with the help of the database the matching and recognition is done and sign image is displayed for the particular text. The user can translate single alphabet or word into sign image.

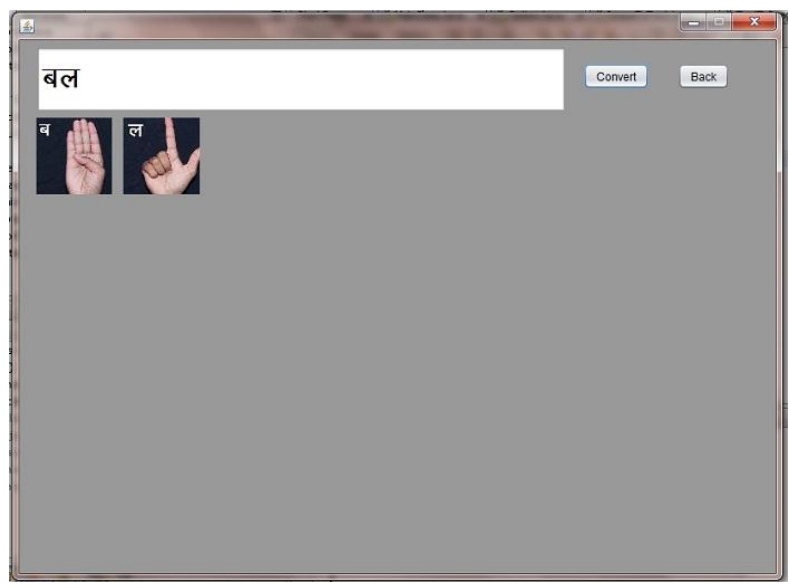

Figure 6.2 Translation of text to sign language in offline.

After getting the correct knowledge related to sign language user will be ready to work on the recognition of sign language through web-camera. During the recognition of sign language through web-cam user needs to perform proper sign in front of the camera. Better results can be obtained by performing correct and accurate signs done by the signer in front of webcam. During translation of sign language to text initially input image is translated to gray scale image such as given below: 


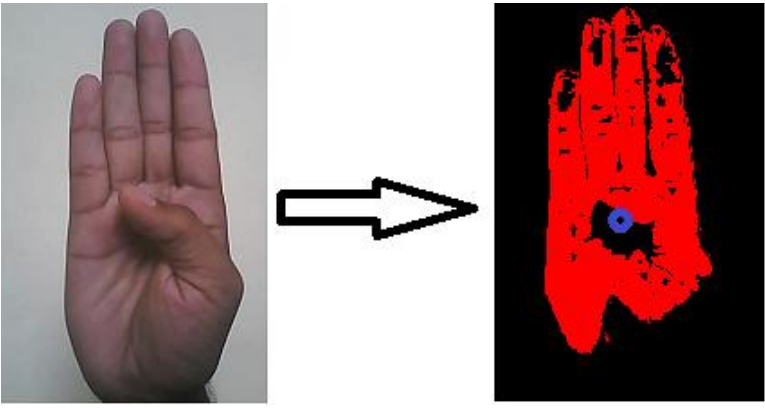

Figure 6.3 Gray scale of input image with center of gravity

The system process input image and matched with database and displays the correct text for that image. During translation resizing of image, color based extraction, noise reduction, calculating center of gravity, translation into binary image and comparison with database is done. And then the correct output is recognizes and displayed on the screen. Snapshot given below shows the translation of sign language to text.

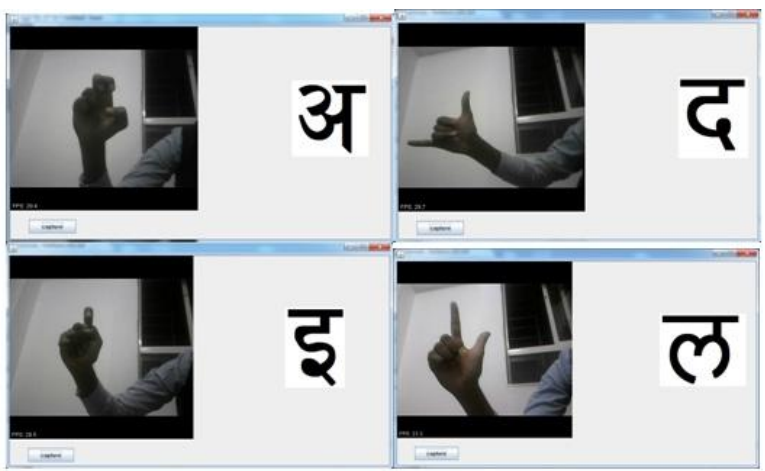

Figure 6.4 Translation of sign language to text (Webcam)

The table given below shows the comparison of the different sign language recognition system. That gives hit/miss ratio, number of signs, and techniques used for recognition.

Table 6.1: Comparison of different recognition methods

\begin{tabular}{|c|c|c|c|c|}
\hline & $\begin{array}{l}\text { Hidden } \\
\text { Markov } \\
\text { model } \\
\text { (HMM) }\end{array}$ & $\begin{array}{c}\text { Euclidean } \\
\text { Distance }\end{array}$ & $\begin{array}{c}\text { Sensor } \\
\text { Based }\end{array}$ & $\begin{array}{c}\text { Proposed } \\
\text { System }\end{array}$ \\
\hline $\begin{array}{c}\text { Hit/ Miss } \\
\text { operation }\end{array}$ & $82 \%$ & $80 \%$ & $93 \%$ & $85 \%$ \\
\hline Signs & 20 & 24 & 10 & 43 \\
\hline $\begin{array}{c}\text { Techniqu } \\
\text { es }\end{array}$ & $\begin{array}{c}\text { Finger- } \\
\text { print } \\
\text { tracking, } \\
\text { Feature } \\
\text { Extraction }\end{array}$ & $\begin{array}{c}\text { Hand } \\
\text { Gloves, } \\
\text { Cyber } \\
\text { Gloves }\end{array}$ & $\begin{array}{c}\text { Segmentat } \\
\text { ion } \\
\text { camera, }\end{array}$ \\
\hline
\end{tabular}

The hit/miss ratio is depending on the alphabets recognized from the total number of alphabets used by the system for the translation. As the number of signs increases the complexity of system increases and recognition rate decrease and along with that overall performance of system degrades. The technique defines the number of ways used for the recognition of sign language. With the better technique the better results are obtained.
The table and the chart given below tell us the recognition rate in the form of percentage. Some selected samples are taken for the generation of graph that shows both best case as well as worst case.

\begin{tabular}{|c|c|c|}
\hline Sr. No. & Alphabet & Detection Rate \\
\hline 1. & $\mathrm{~A}$ & $100 \%$ \\
\hline 2. & $\mathrm{Ai}$ & $100 \%$ \\
\hline 3. & $\mathrm{Na}$ & $60 \%$ \\
\hline 4. & $\mathrm{Kha}$ & $80 \%$ \\
\hline 5. & $\mathrm{O}$ & $30 \%$ \\
\hline 6. & $\mathrm{La}$ & $100 \%$ \\
\hline
\end{tabular}

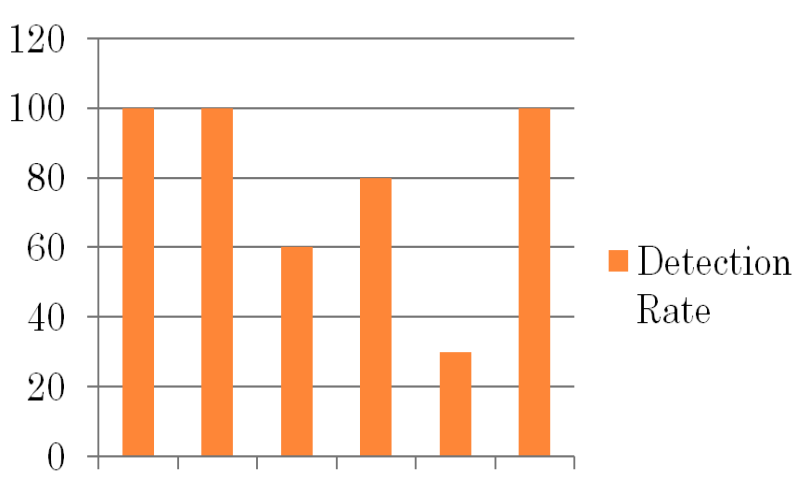

A Ai Na Kha O La

Based on the above table the graph given below shows the detection rate for selected input sign language images. The proposed system is designed in JAVA and obtains the better results as compare to other system without using any device such as sensors or data gloves, or cyber gloves. The system will work correctly and efficiently if signer performs correct and accurate signs in front of camera. For the offline detection and recognition of sign language system gives $100 \%$ results. But because of different signers having different hand size and the method of performing signs in front of camera the system gives maximum expected results.

\section{CONCLUSION AND FUTURE WORK}

Deaf sign users are purely depends on the sign language interpreter for communication. So they cannot be relying on the interpreter in each and every day. Also the cost of the interpreter is very high which cannot be possible for each deaf sign user for each day. This system will help deaf sign user in improving their quality of life significantly. With the help of this system deaf person can be signer independent. The vocal people from society can also learn the sign language and contribute to communicate with the deaf people. The offline mode recognition will helps to learn sign language and gives $100 \%$ results. The offline detection of sign language will work based on the predefined database. The different images were tested and result found that new technique of classification gives $90 \%$ accuracy. The proposed system was implemented in JAVA. The system was designed for recognition of Marathi sign language. And system was capable of handling static alphabets. We have tried to increase the recognition rate of the previous work. We have performed experiments only with the static hand gesture recognition. 
In future work we are trying to improve accuracy of the system. Also we will try for the recognition of dynamic hand gesture along with the facial expressions and made the deaf person independent of interpreter.

\section{ACKNOWLEDGMENTS}

The authors would like to thank Chairman Groups and Management and the Director/Principal Dr.Uttam Kalawane, Colleague of the Department of Computer Engineering and Colleagues of the varies Department the D.Y.Patil School of Engineering and Technology, Pune Dist. Pune Maharashtra, India, for their support, suggestions and encouragement

\section{REFERENCES}

[1] Jung-BaeKim,Kwang-Hyun Park,Won_Chul Bang and Z.Zenn Bien Div.OfEE,Dept of EECS,KAIST, Daejeon, Republic of Korea.Continuous Korean sign language recognition using gesture segmentation and HMM. IEEE-2010.

[2] Venkatraman.S and T.V. Padmavathi, " Speech For The Disabled" , Proceedings of the International MultiConference of Engineers and Computer Scientists 2009 Vol I IMECS 2009, March 18 - 20, 2009.

[3] Gaurav N. Pradhan, Chuanjun Li, Balakrishnan Prabhakaran, "Hand Gesture-based Computing for Hearing and Speech Impaired", IEEE Multimedia Magazine, Vol. 15, No. 2, pp. 20-27, April-June 2008.

[4] Aleemkhalid ,Ali M, M. Usman, S. Mumtaz, Yousuf "BolthayHaath - Paskistan sign Language Recgnition" CSIDC 2005.

[5] Kadous, Waleed "GRASP: Recognition of Australian sign language using Instrumented gloves", Australia, October 1995,pp. 1-2,4-8.

[6] D. E. Pearson and J. P. Sumner, "An experimental visual telephone system for the deaf," J . Roy. Television Society vol. 16, no. 2.pp. 6-10, 1976.

[7] Guitarte Perez, J.F.; Frangi, A.F.; Lleida Solano, E.; Lukas, K. "Lip Reading for Robust Speech Recognition on Embedded Devices" Volume 1, March 18-23, 2005 PP473 - 476 .

[8] DONPEARSON "Visual Communication Systems for the Deaf" IEEE transactions on communications, vol. com-29, no. 12, December 1981.

[9] T. Masuko, K. Tokuda, T. Kobayashi, and S. Imai, "Speech synthesis using HMMs with dynamic features," in Proc. ZCASSP-96, May 1996, pp. 389-392.

[10] SantoshKumar,S.A.; Ramasubramanian, V." Automatic Language Identification Using Ergodic HMM" Acoustics, Speech, and Signal Processing, 2005.Proceedings. (ICASSP'05).IEEE International Conference Vol1,March18-23,2005Page(s):609-612.

[11] honggangwang, ming c. leu and cemiloz, "American Sign Language recognition using multidimensional Hidden Markov Models. Journal of information science and engineering 22, 1109-1123(2006) .Department of Industrial Engineering Purdue University.

[12] Development of a new "Sign Writer" program Daniel Thomas
[13] CULSHAW MURRY (1983) "It Will Soon Be Dark. The situation of disabled in India.Delhi lithouse publications.

[14] DeshmukhDilip (1994) the status of sign language in deaf education in India.Signpost. Newsletter of International Sign Language Association 7(1) 49-52.

[15] Starner, T. (1995). Visual recognition of American Sign Language using hidden Markov models.Master's thesis, Massachusetts Institutes of Technology.

[16] Duda, R. O., \& Hart, P. E. (1972). Use of Hough transformation to detect lines and curves in pictures. Communications of the ACM, 15, 11-15.

[17] KouroshKhoshelham. Extending the use of Hough Transform to detect 3D objects in laser range data. ISPRS Workshop on Laser Scanning 2007 and SilviLaser2007, Espoo, September 12- 14,2007,Finland.

[18] Ong, S., and Ranganath, S. (2005) Automatic sign language analysis: a survey and the future beyond lexical meaning. IEEE Transactions on Pattern Analysis and Machine Intelligence, 27(6).

[19] Symeoinidis, k. (2000). "Hand gesture recognitionusing neural networks." Master's thesis, University of Surrey.

[20] Vamplew,p.(1996). Recognition of sign language using neural networks PhD Thesis, Department of computer Science, and University of Tasmania.

[21] Watson, R. (1993). A survey of gesture recognition techniques.Technical report TCD-CS-93-11, Department of computer Science, Trinty College Dublin.

[22] C.W. ong and surendra and ranganath Automatic sign language analysis: A survey and future beyond lexial meaning sylvie IEEE transactions on pattern analysis and machine intelligence, Vol27,No.6,June 2006

[23] S. Saengsri, V. Niennattrakul, and C.A. Ratanamahatana, "TFRS: Thai Finger-Spelling Sign Language Recognition System", IEEE, 2012, pp. 457-462.

[24] J. H. Kim, N. D. Thang, and T. S. Kim, "3-D Hand Motion Tracking and Gesture Recognition Using a Data Glove", IEEE International Symposium on Industrial Electronics (ISIE), July 5-8, 2009, Seoul Olympic Parktel, Seoul, Korea, pp. 1013-1018.

[25] J. Weissmann and R. Salomon, "Gesture Recognition for Virtual Reality Applications Using Data Gloves and Neural Networks", IEEE, 1999, pp. 2043-2046

[26] M. V. Lamar, S. Bhuiyan, and A. Iwata, "Hand Alphabet Recognition Using Morphological PCA and Neural Networks", IEEE, 1999, pp. 2839-2844.

[27] T. Kapuscinski and M. Wysocki, "Hand Gesture Recognition for Man-Machine interaction", Second Workshop on Robot Motion and Control, October 1820, 2001, pp. 91-96.

[28] M. Pahlevanzadeh, M. Vafadoost, and M. Shahnazi, "Sign Language Recognition", IEEE, 2007.

[29] J. Rekha, J. Bhattacharya, and S. Majumder, "Shape, Texture and Local Movement Hand Gesture Features 
for Indian Sign Language Recognition”, IEEE, 2011, pp. 30-35.

[30] Y. Wu and T.S. Huang, "Vision-based gesture recognition: A review," Lecture Notes Comput. Sci., vol. 1739, pp. 103-115, 1999.

\section{AUTHOR 'S PROFILE}

Mr. Amitkumar Shinde received Bachelor of Engineering degree in Computer Science \& Engineering in 2012 and now pursuing Post Graduation (M.E.) in department of Computer
Engineering from Dr. D.Y.Patil School of Engineering and Technology in current academic year 2014-15. He is now studying for the domain Image Processing as research purpose on Sign Language recognition for Deaf Sign User in his academic year.

Prof. Ramesh Kagalkar (Ph.D. Scholar) $\mathrm{He}$ is now assistant professor in department of computer engineering Dr. D. Y. Patil School of Engineering and Technology Lohegaon , Pune. He is now in the research field of area of Image Processing. 